\title{
Teleparallel origin of the Fierz picture for spin-2 particle
}

\author{
Yu. N. Obukhov* and J. G. Pereira \\ Instituto de Física Teórica, Universidade Estadual Paulista, Rua Pamplona 145, 01405-900 São Paulo, Brazil
}

(Received 31 October 2002; published 26 February 2003)

\begin{abstract}
A new approach to the description of a spin-2 particle in flat and curved spacetime is developed on the basis of the teleparallel gravity theory. We show that such an approach is in fact a true and natural framework for the Fierz representation proposed recently by Novello and Neves. More specifically, we demonstrate how the teleparallel theory fixes uniquely the structure of the Fierz tensor, discover the transparent origin of the gauge symmetry of the spin-2 model, and derive the linearized Einstein operator from the fundamental identity of the teleparallel gravity. In order to cope with the consistency problem on the curved spacetime, similarly to the usual Riemannian approach, one needs to include the nonminimal (torsion dependent) coupling terms.
\end{abstract}

DOI: 10.1103/PhysRevD.67.044008

PACS number(s): 04.50.+h, 03.50.-z, 04.20.Cv, 11.10.Ef

\section{INTRODUCTION}

A consistent description of higher (greater than 1) spin fields interacting with external classical (electromagnetic and gravitational) fields represents a nontrivial problem which has not been completely solved until now. Earlier analyses of this problem can be found in Refs. [1,2], and a more recent discussion, as well as a list of basic references, is given in Ref. [3]. Recently, a new approach based on the so called Fierz theory of the spin-2 particle in flat and curved spacetime has been developed by Novello and Neves [4]. In this work, using Fierz variables, the consistency problem of a massive spin-2 field in a curved spacetime has been studied, with the results indicating that the Fierz representation seems to be more appropriate to deal with the coupling of a spin-2 field to gravity.

Now, as is well known, curvature and torsion are able to provide each one equivalent description of the gravitational interaction. In fact, according to general relativity, curvature is used to geometrize spacetime, and in this way successfully describe the gravitational interaction. Teleparallelism, on the other hand, attributes gravitation to torsion, but in this case torsion accounts for gravitation not by geometrizing the interaction, but by acting as a force. This means that, in the teleparallel equivalent of general relativity, there are no geodesics, but force equations quite analogous to the Lorentz force equation of electrodynamics [5]. Gravitational interaction, thus, can be described alternatively in terms of curvature, as is usually done in general relativity, or in terms of torsion, in which case we have the so called teleparallel gravity.

An important point of teleparallel gravity is that it corresponds to a gauge theory for the translation group. Due to the peculiar character of translations, any gauge theory including these transformations will differ from the usual internal gauge models in many ways, the most significant being the presence of a tetrad field. On the other hand, a tetrad field can naturally be used to define a linear Weitzenböck connection, which is a connection presenting torsion, but no curva-

\footnotetext{
*Permanent address: Department of Theoretical Physics, Moscow State University, 117234 Moscow, Russia.
}

ture. A tetrad field can also be used to define a Riemannian metric, in terms of which a Levi-Civita connection is constructed. As is well known, it is a connection presenting curvature, but no torsion. It is important to keep in mind that torsion and curvature are properties of a connection [6], and many different connections can be defined on the same manifold. Therefore one can say that the presence of a nontrivial tetrad induces both a teleparallel and a Riemannian structures in spacetime. The first is related to the Weitzenböck and the second to the Levi-Civita connection.

The reason for gravitation to present two equivalent descriptions is related to a quite peculiar property of gravitation, the so called universality. Let us explore this point in more detail. As with any other interaction, gravitation presents a description in terms of gauge theory. In fact, as mentioned above, teleparallel gravity is a gauge theory for the translation group, with contortion playing the role of force. On the other hand, universality of gravitation means that all particles feel gravity the same. In other words, particles with different masses feel a different gravitational force in such a way that all these particles acquire the same acceleration. As a consequence of this property, it turns out to be possible to describe gravitation not as a force, but as a deformation of spacetime. More precisely, according to this view, a gravitational field is supposed to produce a curvature in spacetime, the gravitational interaction being achieved in this case by letting test particles to follow the geodesics of spacetime. This is the approach used by the general relativity description of gravitation. It is important to notice that only an interaction presenting the universality property can be described by a geometrization of spacetime.

Instead of using the general relativity approach, the basic purpose of this paper will be to use the teleparallel approach to analyze the problem of a spin- 2 field coupled to gravity. The teleparallel approach will be used to describe both the spin-2 field, and the gravitational background field. The basic conclusion will be that the Fierz picture constructed in Ref. [4] is naturally present in the teleparallel construction. In this sense, we can say that the teleparallel equivalent of general relativity appears to be a natural framework to deal with the spin-2 theory. In fact, the small perturbations of the tetrad field are shown to reproduce correctly the behavior of the spin-2 field on the flat Minkowski spacetime. The antisym- 
metric piece of the tetrad turns out to be redundant, although taking into account its explicit contribution makes the underlying gauge symmetry more transparent. The generalization for the presence of gravitation is straightforward, and it represents an alternative way to describe the spin- 2 particle interacting with an external gravitational field.

\section{TELEPARALLEL GRAVITY: BASIC FACTS}

The general structure of teleparallel gravity is presented in detail in Refs. [5,7-10]. In this section we summarize the fundamentals of this theory. In short, teleparallel approach can be understood as a gauge theory of the spacetime translation group. Using the Greek alphabet to denote spacetime indices, and the Latin alphabet to denote the local frame components, the corresponding gauge potential is represented by the nontrivial part of the tetrad field $h^{a}{ }_{\mu}$. This tetrad gives rise to the so called Weitzenböck connection

$$
\Gamma_{\mu \nu}^{\rho}=h_{a}{ }^{\rho} \partial_{\nu} h_{\mu}^{a},
$$

which introduces the distant parallelism on a fourdimensional spacetime manifold. It is a connection that presents torsion, but not curvature. Its torsion

$$
T_{\mu \nu}^{\rho}=\Gamma_{\nu \mu}^{\rho}-\Gamma_{\mu \nu}^{\rho}
$$

plays the role of the translational gauge field strength. The Weitzenböck connection can be conveniently decomposed into the Riemannian and the post-Riemannian pieces

$$
\Gamma_{\mu \nu}^{\rho}=\widetilde{\Gamma}_{\mu \nu}^{\rho}+K^{\rho}{ }_{\mu \nu},
$$

where $\widetilde{\Gamma}_{\mu \nu}^{\rho}=\frac{1}{2} g^{\rho \sigma}\left(\partial_{\mu} g_{\nu \sigma}+\partial_{\nu} g_{\mu \sigma}-\partial_{\sigma} g_{\mu \nu}\right)$ is the Christoffel symbol constructed from the spacetime metric $g_{\mu \nu}$ $=h^{a}{ }_{\mu} h^{b}{ }_{\nu} \eta_{a b}$ and

$$
K_{\mu \nu}^{\rho}=\frac{1}{2}\left(T_{\mu}{ }^{\rho}{ }_{\nu}+T_{\nu}{ }^{\rho}{ }_{\mu}-T^{\rho}{ }_{\mu \nu}\right)
$$

is the contortion tensor. Correspondingly, all other geometrical and physical objects and operations constructed with the help of the Riemannian connection $\widetilde{\Gamma}^{\rho}{ }_{\mu \nu}$ will be denote with a tilde.

The gauge gravitational field Lagrangian reads

$$
\mathcal{L}_{G}=\frac{c^{4}}{16 \pi G} h S^{\rho \mu \nu} T_{\rho \mu \nu},
$$

where $h=\operatorname{det}\left(h_{\mu}^{a}\right)$ and

$$
S^{\rho \mu \nu}=-S^{\rho \nu \mu}:=\frac{1}{2}\left[K^{\mu \nu \rho}-g^{\rho \nu} T_{\sigma}^{\sigma \mu}+g^{\rho \mu} T_{\sigma}^{\sigma \nu}\right]
$$

is a tensor written in terms of the Weitzenböck connection only. Inverting this equation, we obtain torsion in terms of the above tensor:

$$
T^{\rho}{ }_{\mu \nu}=2\left(S_{\mu}{ }^{\rho}{ }_{\nu}-S_{\nu}{ }^{\rho}{ }_{\mu}\right)+\delta_{\mu}^{\rho} S_{\nu \sigma}^{\sigma}-\delta_{\nu}^{\rho} S_{\mu \sigma}^{\sigma} .
$$

The Lagrangian (2.5) describes what is commonly known as the teleparallel equivalent of Einstein's general relativity theory. Performing a variation with respect to the tetrad, we find the teleparallel version of the gravitational field equation

$$
\partial_{\sigma}\left(h S_{\lambda}^{\rho \sigma}\right)-\frac{4 \pi G}{c^{4}}\left(h t_{\lambda}^{\rho}\right)=0,
$$

where

$$
h t_{\lambda}{ }^{\rho}=\frac{c^{4} h}{4 \pi G} S_{\mu}{ }^{\nu \rho} \Gamma^{\mu}{ }_{\nu \lambda}-\delta_{\lambda}{ }^{\rho} \mathcal{L}_{G}
$$

is the energy-momentum (pseudo) tensor of the gravitational field. This equation is known to be equivalent to the Einstein's equation of general relativity. It is important to notice that the left-hand side of the field equation (2.8) can be rewritten as the usual left-hand side of Einstein equations

$$
\partial_{\sigma}\left(h S_{\lambda}^{\rho \sigma}\right)-\frac{4 \pi G}{c^{4}}\left(h t_{\lambda}^{\rho}\right) \equiv \frac{h}{2}\left(\widetilde{R}_{\lambda}^{\rho}-\frac{1}{2} \delta_{\lambda}^{\rho} \widetilde{R}\right),
$$

which then provides an easy proof of the Lemma 2 of Ref. [4]. As the source of both field equations is the symmetric energy-momentum tensor, the equivalence alluded to above holds also in the presence of matter [11]. It is worth noticing that the teleparallel field equation has the same structure of the Yang-Mills equation, which is consistent with the fact that teleparallel gravity corresponds to a gauge theory. We see in this way that the teleparallel approach to gravitation is more closely related to field theory than the general relativity approach.

\section{LINEARIZED THEORY}

The trivial tetrad $h^{a}{ }_{\mu}=\delta^{a}{ }_{\mu}$ describes the flat geometry, for which the metric has the diagonal Minkowski form $g_{\mu \nu}$ $=\eta_{\mu \nu}=\operatorname{diag}(+1,-1,-1,-1)$. Let us then expand the tetrad field around the flat background as follows:

$$
h^{a}{ }_{\mu}=\delta^{a}{ }_{\mu}+u^{a}{ }_{\mu} .
$$

The Weitzenböck connection reads, correspondingly,

$$
\Gamma_{\mu \nu}^{\rho}=\partial_{\nu} u_{\mu}^{\rho},
$$

where $u^{\rho}{ }_{\mu}=\delta_{a}{ }^{\rho} u^{a}{ }_{\mu}$. As a result, the torsion and its trace are, respectively,

$$
T_{\mu \nu}^{\rho}=\partial_{\mu} u^{\rho}{ }_{\nu}-\partial_{\nu} u_{\mu}^{\rho}, \quad T_{\mu \rho}^{\rho}=\partial_{\mu} u-\partial_{\rho} u^{\rho}{ }_{\mu},
$$

with $u=u_{\rho}^{\rho}$. Decomposing the perturbation tensor $u_{\mu \nu}$ into the symmetric and antisymmetric pieces

$$
u_{\mu \nu}=\phi_{\mu \nu}+a_{\mu \nu}
$$

with

$$
\phi_{\mu \nu}:=u_{(\mu \nu)} \quad \text { and } \quad a_{\mu \nu}:=u_{[\mu \nu]},
$$

we compute immediately the contortion tensor

$$
K_{\mu \nu}^{\rho}=\partial^{\rho} \phi_{\mu \nu}-\partial_{\mu} \phi_{\nu}^{\rho}+\partial_{\nu} a^{\rho}{ }_{\mu} .
$$



obtain

Substituting now the above expressions into Eq. (2.6), we

$$
\begin{aligned}
S^{\rho \mu \nu}= & \frac{1}{2}\left[\partial^{\mu} \phi^{\nu \rho}-\partial^{\nu} \phi^{\mu \rho}-g^{\rho \nu}\left(\partial^{\mu} \phi-\partial_{\sigma} \phi^{\sigma \mu}\right)\right. \\
& +g^{\rho \mu}\left(\partial^{\nu} \phi-\partial_{\sigma} \phi^{\sigma \nu}\right) \\
& \left.+\partial^{\rho} a^{\mu \nu}+g^{\rho \nu} \partial_{\sigma} a^{\sigma \mu}-g^{\rho \mu} \partial_{\sigma} a^{\sigma \nu}\right] .
\end{aligned}
$$

Comparison with Ref. [4] shows that the first two lines are nothing but the Fierz tensor $F^{\mu \nu \rho}=-F^{\nu \mu \rho}$ introduced by Novello and Neves. More specifically,

$$
S^{\rho \mu \nu}=-F^{\mu \nu \rho}+\frac{1}{2}\left(\partial^{\rho} a^{\mu \nu}+g^{\rho \nu} \partial_{\sigma} a^{\sigma \mu}-g^{\rho \mu} \partial_{\sigma} a^{\sigma \nu}\right) .
$$

Since we have identically

$$
\partial_{\mu}\left(\partial^{\rho} a^{\mu \nu}+g^{\rho \nu} \partial_{\sigma} a^{\sigma \mu}-g^{\rho \mu} \partial_{\sigma} a^{\sigma \nu}\right) \equiv 0,
$$

the last term drops out completely from the linearized gravitational field equations (2.8), which then reads

$$
\partial_{\sigma} S_{\lambda}^{\sigma \rho}=0 .
$$

This equation yields the correct dynamics of the spin-2 particle in flat spacetime, as it is easily seen from the identity (2.10). Indeed, substituting the expansion (3.1) into it, we find that

$$
\partial_{\sigma} S_{\lambda}{ }^{\sigma \rho} \equiv \frac{1}{2} \widetilde{G}_{\lambda}^{\mathrm{L}}{ }^{\rho},
$$

where the left-hand side represents the linearized Einstein tensor

$$
\begin{aligned}
\widetilde{G}_{\mu \nu}^{\mathrm{L}}= & \square\left(\eta_{\mu \nu} \phi-\phi_{\mu \nu}\right)-\partial_{\mu} \partial_{\nu} \phi-\eta_{\mu \nu} \partial_{\alpha} \partial_{\beta} \phi^{\alpha \beta}+\partial_{\mu} \partial_{\lambda} \phi^{\lambda}{ }_{\nu} \\
& +\partial_{\nu} \partial_{\lambda} \phi^{\lambda}{ }_{\mu} .
\end{aligned}
$$

The identity (2.10) plays the fundamental role in the teleparallel theory, since it underlies the proof of the equivalence of Einstein's gravity and the teleparallel gravity. Now, as a byproduct of this identity we have straightforwardly derived the Lemma 2 of Ref. [4].

It is interesting to notice that the teleparallel Lagrangian (2.5) can be rewritten as

$$
\mathcal{L}_{G}=\frac{c^{4}}{8 \pi G} h\left(S^{\rho \mu \nu} S_{\rho \mu \nu}-S^{\sigma \mu}{ }_{\sigma} S^{\rho}{ }_{\mu \rho}-3 S^{\rho \mu \nu} S_{[\rho \mu \nu]}\right) .
$$

Inserting Eq. (3.7) here, we can verify that the antisymmetric field $a_{\mu \nu}$ drops out completely, in accordance with the analysis of the linearized field equations. This observation shows that, as a matter of fact, the antisymmetric field does not have physical importance. Indeed, one can show quite generally that, by means of a local Lorentz transformation, it is always possible to choose a frame in which the tetrad matrix is symmetric [12-14]. In such a frame, the field $a_{\mu \nu}$ vanishes, and as a result the tensor $S^{\rho \mu \nu}$ coincides (up to a sign) with the Fierz tensor introduced in Ref. [4].
However, there is a certain reason to keep $a_{\mu \nu}$ nontrivial. In particular, we can verify the covariance of the linearized formalism with respect to "general coordinate" spacetime transformations. The demonstration of this property in Ref. [4] is rather long and not very transparent. In contrast, here it is sufficient to notice that the tensor (3.7) is explicitly invariant under the gauge transformations

$$
\begin{aligned}
& \phi_{\mu \nu} \rightarrow \phi_{\mu \nu}+\partial_{\mu} \Lambda_{\nu}+\partial_{\nu} \Lambda_{\mu}, \\
& a_{\mu \nu} \rightarrow a_{\mu \nu}-\partial_{\mu} \Lambda_{\nu}+\partial_{\nu} \Lambda_{\mu} .
\end{aligned}
$$

The proof is straightforward: One just needs to substitute these formulas into Eq. (3.7). The original perturbation field (3.4) transforms as $u^{\mu}{ }_{\nu} \rightarrow u^{\mu}{ }_{\nu}+2 \partial_{\nu} \Lambda^{\mu}$, in complete agreement with the geometrical meaning of the tetrad.

\section{SPIN-2 FIELD IN THE PRESENCE OF GRAVITATION}

In order to construct the theory for a spin- 2 particle on a curved spacetime, instead of the expansion (3.1), we consider the tetrad expansion

$$
h^{a}{ }_{\mu}=\bar{h}_{\mu}^{a}+u_{\mu}^{a}
$$

around the nontrivial classical background $\bar{h}^{a}{ }_{\mu}$. Such an approach is analogous to the treatment of a consistent spin-2 model as a first order perturbation of the general relativity theory [2]. We will denote with an overline every other background objects and operations. In order to simplify the computations, in contrast to the above described flat-space discussion, we will choose the symmetric gauge [12-14] from the very beginning. Then, we have the symmetric tensor field

$$
\phi_{\nu}^{\mu}:=\bar{h}_{a}{ }^{\mu} u_{\nu}^{a}, \quad \phi_{\mu \nu}=\phi_{\nu \mu},
$$

where $\bar{h}_{a}{ }^{\mu}$ is the inverse background tetrad $\bar{h}_{a}{ }^{\mu} \bar{h}^{a}{ }_{\nu}=\delta_{\nu}^{\mu}$. From now on, the Greek indices will be raised and lowered with the help of the background metric $\bar{g}_{\mu \nu}=\bar{h}^{a}{ }_{\mu} \bar{h}^{b}{ }_{\nu} \eta_{a b}$.

Substituting the expansion (4.1) into the Weitzenböck connection (2.1), up to first order in $\phi^{\rho}{ }_{\mu}$, we find

$$
\Gamma_{\mu \nu}^{\rho}=\bar{\Gamma}_{\mu \nu}^{\rho}+\bar{\nabla}_{\nu} \phi_{\mu}^{\rho},
$$

where $\bar{\Gamma}_{\mu \nu}^{\rho}=\bar{h}_{a}{ }^{\rho} \partial_{\nu} \bar{h}^{a}{ }_{\mu}$ is the background teleparallel connection, and $\bar{\nabla}_{\nu}$ is the covariant derivative in the connection $\bar{\Gamma}^{\rho}{ }_{\mu \nu}$. As a result, we obtain for the torsion

$$
T^{\rho}{ }_{\mu \nu}=\bar{T}_{\mu \nu}^{\rho}+\bar{\nabla}_{\mu} \phi^{\rho}{ }_{\nu}-\bar{\nabla}_{\nu} \phi^{\rho}{ }_{\mu} .
$$

Using this expression in Eqs. (2.6) and (3.12), we straightforwardly obtain the kinetic term for the spin-2 field Lagrangian in the presence of gravitation

$$
\mathcal{L}_{G}=\frac{c^{4}}{8 \pi G} \bar{h}\left(F^{\rho \mu \nu} F_{\rho \mu \nu}-F_{\sigma}^{\sigma \mu} F^{\rho}{ }_{\mu \rho}\right)=\frac{c^{4}}{8 \pi G} \bar{h} F^{\rho \mu \nu} \bar{\nabla}_{\nu} \phi_{\mu \rho} .
$$

Here, $F^{\rho \mu \nu}$ is the covariant generalization of the Fierz tensor

$$
\begin{aligned}
F^{\rho \mu \nu}= & \frac{1}{2}\left[\bar{\nabla}^{\nu} \phi^{\mu \rho}-\bar{\nabla}^{\mu} \phi^{\nu \rho}+g^{\rho \nu}\left(\bar{\nabla}^{\mu} \phi-\bar{\nabla}_{\sigma} \phi^{\sigma \mu}\right)\right. \\
& \left.-g^{\rho \mu}\left(\bar{\nabla}^{\nu} \phi-\bar{\nabla}_{\sigma} \phi^{\sigma \nu}\right)\right] .
\end{aligned}
$$


The main difference of the spin-2 model (4.5) from the theory studied in Ref. [4] is that the covariant derivatives in Eq. (4.6) are defined with the help of the teleparallel connection, and not with the help of the Riemannian (Christoffel) connection. We thus obtain an alternative way to describe a spin-2 particle in the presence of gravitation. The resulting theory is obviously generally covariant.

As is well known [1-4], the higher spin theories are generally inconsistent in the presence of the electromagnetic and/or gravitational field. Technically, this amounts to the nonvanishing covariant divergence of the corresponding field operator which, in turn, is related to the fact that the covariant derivative has a nontrivial commutator proportional to the curvature. The consistency conditions, derived for the higher spin fields, place strong restrictions on the spacetime curvature which are not fulfilled, in general.

In the teleparallel gravity, the spacetime curvature is zero, whereas the commutator of the covariant derivatives reads

$$
\left[\nabla_{\mu}, \nabla_{\nu}\right]=-T_{\mu \nu}^{\lambda} \nabla_{\lambda} .
$$

Accordingly, the consistency condition for the theory (4.5) will be not algebraic, as in the usual formulation, but differential instead: $\bar{T}_{\mu \nu}^{\lambda} \bar{\nabla}_{\lambda} F^{\rho \mu \nu}=0$.

If we demand that the consistency conditions should be satisfied for all field configurations, we then have to conclude that the torsion should vanish. This observation agrees with the general analysis of the higher spin theories on the Riemann-Cartan spacetime [15]. A possible way to avoid the consistency problem is to include nonminimal coupling terms in the Lagrangian [2,3]. Since the curvature is zero, the nonminimal terms may involve only the torsion tensor. The latter, being of the third rank, necessarily involves the derivative for the construction of the invariant contractions. It is straightforward to see that, adding the nonminimal interaction Lagrangian

$$
\begin{aligned}
\mathcal{L}_{\text {non }}= & \frac{c^{4}}{8 \pi G} \bar{h}\left[\phi_{\alpha \lambda}\left(K^{\lambda}{ }_{\mu \nu}-T^{\lambda}{ }_{\mu \nu}\right) \widetilde{\nabla}^{\nu} \phi^{\mu \alpha}-\phi^{\alpha \mu} T_{\lambda \mu \nu} \widetilde{\nabla}_{\alpha} \phi^{\lambda \nu}\right. \\
& -\phi^{\lambda \nu} T_{\lambda \mu \nu}\left(\widetilde{\nabla}^{\mu} \phi-\widetilde{\nabla}_{\alpha} \phi^{\alpha \mu}\right) \\
& \left.+\phi^{\mu \nu} T^{\lambda}{ }_{\mu \lambda}\left(\widetilde{\nabla}_{\nu} \phi-\widetilde{\nabla}^{\alpha} \phi_{\alpha \nu}\right)\right]
\end{aligned}
$$

to the Lagrangian (4.5) yields the Riemannian Fierz Lagrangian of Ref. [4]. This then allows us to solve the consistency problem.

The theory of massive spin-2 particle $[2,3]$ was satisfactorily formulated within the framework of the Fierz approach [4]. Our results also admit a direct generalization to the nontrivial mass which we though do not discuss here since it follows along the same lines as in Ref. [4].

\section{DISCUSSION AND CONCLUSIONS}

Novello and Neves [4] have demonstrated that the Fierz representation for the spin-2 theory has a number of advantages as compared to the alternative approaches (such as the nonambiguity of the order of derivatives and the equivalence to the nonminimal curvature Einstein representation with the fixed coefficients of additional terms [2]). Here, we have shown that the Fierz representation can be naturally understood on the basis of the teleparallel gravity. In particular, we find that (i) the structure of the Fierz tensor defined in Ref. [4] through an ad hoc procedure is unambiguously fixed by the teleparallel theory, (ii) the gauge symmetry (3.13),(3.14) underlying the corresponding spin-2 freedom, is manifested straightforwardly, (iii) the linearized Einstein operator arises immediately as a consequence of the fundamental identity (2.10) of the teleparallel theory. It is worthwhile to note that the antisymmetric piece of the tetrad field, while being dynamically redundant, plays a significant role in the formal derivations. A certain disadvantage of our approach is the need of the nonminimal coupling of the type (4.8) to solve the consistency problem on the curved spacetime. This is similar to the observations made within the Riemannian approach [2].

Summarizing, in this paper we have developed a new approach to the description of spin-2 in flat and curved spacetime on the basis of the teleparallel gravity theory. This approach appears to be a true origin for the Fierz representation proposed recently in Ref. [4].

\section{ACKNOWLEDGMENTS}

The work of Y.N.O. was supported by FAPESP. J.G.P. thanks FAPESP and CNPq for partial financial support. We are grateful to Friedrich Hehl for valuable advice.
[1] W. Pauli and M. Fierz, Helv. Phys. Acta 12, 297 (1939); M. Fierz and W. Pauli, Proc. R. Soc. London, Ser. A A173, 211 (1939).

[2] C. Aragone and S. Deser, Nuovo Cimento A 3, 709 (1971); C. Aragone and S. Deser, Nuovo Cimento Soc. Ital. Fis., B 57, 33 (1980).

[3] I. L. Buchbinder, D. M. Gitman, V. A. Krykhtin, and V. D. Pershin, Nucl. Phys. B584, 615 (2000).

[4] M. Novello and R. P. Neves, Class. Quantum Grav. 19, 5335 (2002).

[5] V. C. de Andrade and J. G. Pereira, Phys. Rev. D 56, 4689 (1997).
[6] See, for example, R. Aldrovandi and J. G. Pereira, An Introduction to Geometrical Physics (World Scientific, Singapore, 1995).

[7] K. Hayashi and T. Shirafuji, Phys. Rev. D 19, 3524 (1979).

[8] V. C. de Andrade, L. C. T. Guillen, and J. G. Pereira, Phys. Rev. Lett. 84, 4533 (2000).

[9] V. C. de Andrade, L. C. T. Guillen, and J. G. Pereira, Phys. Rev. D 64, 027502 (2001).

[10] F. W. Hehl, J. D. McCrea, E. W. Mielke, and Y. Ne'eman, Phys. Rep. 258, 1 (1995).

[11] See, for example, M. Blagojević, Gravitation and Gauge Symmetries (IOP Publishing, Bristol, 2002). 
[12] B. S. DeWitt and C. M. DeWitt, Phys. Rev. 87, 116 (1952).

[13] V. I. Ogievetskii and I. V. Polubarinov, Sov. Phys. JETP 21, 1093 (1965).

[14] R. Aldrovandi, S. F. Novaes, and D. Spehler, Gen. Relativ.
Gravit. 26, 409 (1994).

[15] N. H. Barth and S. M. Christensen, J. Phys. A 16, 543 (1983); Yu. N. Obukhov, ibid. 16, 3795 (1983); Yu. N. Obukhov, Nucl. Phys. B212, 237 (1983). 